\title{
The Study of Electron Properties of Carbon Nanotubes Deposited on Highly Orientated Pyrolytical Graphite Using Scanning Probe Spectroscopy
}

\author{
M. Kamiński, M. Wróblewski, M. CȨGiel And B. Susea \\ Institute of Physics, Faculty of Technical Physics \\ Poznań University of Technology, Nieszawska 13a, 60-965 Poznań, Poland
}

In this paper we present experimental investigations of carbon nanotubes deposited on highly orientated pyrolytical graphite using scanning tunneling microscopy and scanning tunneling spectroscopy. The aforementioned methods apart from detailed topographic data provided us with information about local density of state. We also show the $I-V$ and $\mathrm{d} I / \mathrm{d} V$ characteristics, which display the metallic and semiconducting characters of investigated carbon nanotubes. All measurements were taken in the air and at room temperature.

PACS numbers: 68.37.Ef, 73.63.Fg, 73.63.-b

\section{Introduction}

Carbon nanotubes (CNTs) (which are cylindrical objects) may consist of one layer - single-walled nanotubes (SWNTs) or of several layers - multiwalled nanotubes (MWNTs) [1]. The CNT's typical diameter ranges from $1.2 \mathrm{~nm}$ to $2 \mathrm{~nm}$ for SWNT and it is about $25 \mathrm{~nm}$ for MWNT. Their length (height) can amount to several $\mu \mathrm{m}$. CNTs have $s p 2$ hybridization between carbon atoms. One of the basic properties of CNTs, as far as the structure and the electric properties are concerned, is their chirality and earlier mentioned diameter [2-6]. The chiral (or helicity) vector connects two crystallographically equivalent points. The $C$ vector is defined by the $n, m$ symbols (their value can be used in defining the type of the CNT) and the two vectors of hexagonal lattice $a_{1}$ and $a_{2}$, where $C=n a_{1}+m a_{2} \equiv(n, m)$. The values of $n$ and $m$ number have hexagon coordinates on the graphen sheet surface. Such surface, after being rolled up into CNT has identical orientation as the hexagon at the beginning of the system. We can distinguish the characteristic configurations of carbon nanotubes: zigzag $(n, 0)$ and 
armchair $(n, m)$ configuration. The other objects are of the chiral type and have a finite wrapping $\phi$ angle. The $\phi$ angle is included between the zigzag direction $(n, 0)$ and the unit vectors $a_{1}$ and $a_{2}$ (see Fig. 1 ). Due to the six-time rotation of hexagonal cell, $\phi$ angle values are between 0 and 30 degrees [6]. According to Wilder et al. [7]: "All other wrapping angles lead to helicity tubes whose wrapping angle is specified relatively to either the zigzag direction $\theta$ or armchair direction $\phi=30^{\circ}-\theta$. Dashed lines are perpendicular to $C$ and run in the direction of the tube axis indicated by vector $T$. The solid vector $H$ is perpendicular to the armchair direction and specifies the direction of nearest-neighbor hexagon rows, indicated by black dots. The chiral angle $\phi$ is the angle between $T$ and $H$ vectors".

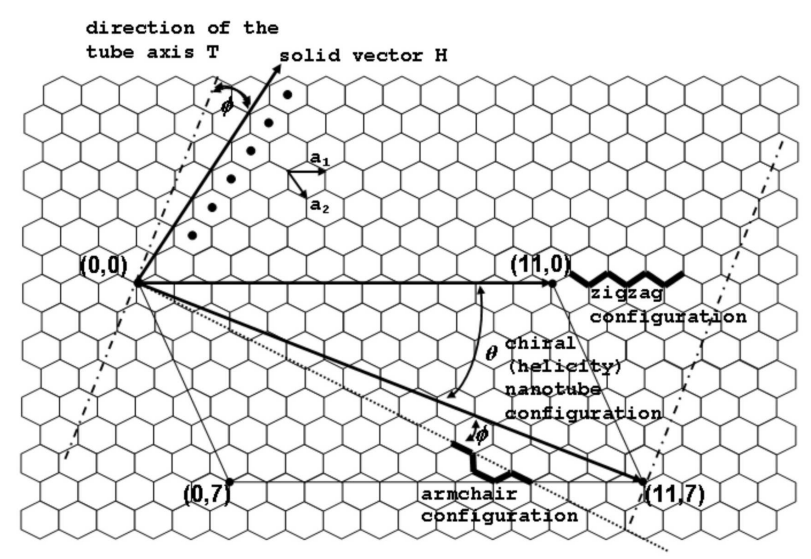

Fig. 1. Division of the graphite layer for nanotubes such as: zigzag, armchair, and chiral, according to Wilder et al. [7].

The theoretical calculations give information that $1 / 3$ of SWNTs have a metallic character while the remaining $2 / 3$ have a semiconducting character. With the following properties of parameters $n$ and $m: n-m=3 q$, where $q$-integer number, SWNT reveals the metallic character [8]. As far as MWNT are concerned, their conducting properties depend on the number of layers which constitute them, as well as the conducting properties of the layers themselves. Additionally, their electrical conductivity decreases with increasing temperature (our measurements were taken at room temperature). The conductivity in CNTs takes place through discrete, strictly defined electron states. In case when the length of the conductivity way is shorter than the free way of electron, then electron transportation has a ballistic character. The electric properties in the CNTs may differ locally. For instance, it was discovered that metallic carbon nanotubes may show the semiconducting character at its ends [9].

In this paper we investigate the conductance of the metallic and semiconducting CNTs. We present clear evidence of the basic properties of CNTs, as far 
as the structure (chirality and diameter) and the electric properties are concerned. Electronic transport properties and local density of state were measured using the scanning tunneling microscopy/scanning tunneling spectroscopy (STM/STS) techniques.

\section{Experiment}

The investigated CNTs came from Center of Advanced European Studies and Research (CAESAR), Bonn. They were located in the substrate collected from arc cathode and suspended in the deionized water. The color of suspension was black (in all likelihood, substrate has not undergone cleaning). High condensation of the deposit in water as well as the size of formed conglomerates prevented us from direct placing of the deposit on the investigated surface. CNT were submerged in highly evaporating solvent-butyl acetate $\left(\mathrm{CH}_{3} \mathrm{COOC}_{4} \mathrm{H}_{9}\right)$. Next, the solution was placed in the ultracentrifuge from 5 up to $10 \mathrm{~min}$. About $0.1 \mathrm{ml}$ of this solution was deposited on the highly orientated pyrolytical graphite (HOPG) surface. To quicken the evaporation the sample was placed in the drier for $8 \mathrm{~min}$ (at temperature of $40-70^{\circ} \mathrm{C}$ ). It was affirmed that deposition of the solution larger than $0.1 \mathrm{ml}$ does not allow obtaining good quality images. It is due to the fact that in such case there are too many CNTs on the investigated surface. On the other hand, using smaller amount of solution than $0.1 \mathrm{ml}$ with maximum scanning surface $850 \mathrm{~nm} \times 850 \mathrm{~nm}$ did not always allow observing CNT. STM has proven to be useful technique in investigating atomic and electronic structure of CNT [10-15]. Research carried out by means of STM and STS are characterized by high sensitivity to all kinds of outward factors - for instance, thermal drift or outward vibrations.

\section{Results and discussion}

\subsection{The $I-V$ and $d I / d V$ characteristics of $S W N T$ and $M W N T$}

Figure 2 presents a single nanotube along which measurements of $I-V$ characteristics are the points marked with capital letters.

Within a low voltage $(<0.5 \mathrm{~V})$ range $I-V$ dependence is linear. Only for higher voltage current it becomes to rise rapidly. It is connected with a higher probability of tunneling transition when the voltage is higher.

The probability of tunneling transition is the highest for electrons on the Fermi level of minus polarized electrode. In the first approach, the tunneling current between two metals is a linear function of polarizing voltage and exponent of tip-sample length. It is true for a small voltage, where $\mathrm{eV}<\phi, \phi-$ thermionic work function. When $\mathrm{eV}>\phi$, the tunneling current depends on the third voltage power which occurs as a result of lowering the effective height barrier. It finds its reflection in $I-V$ characteristics (see Fig. 2). These characteristics consist of linear and non-linear part $\left(\sim V^{3}\right) . I-V$ characteristics have the metallic character and can be described in approximation by the following formula: $I=\alpha\left(V+\gamma V^{3}\right)$, 


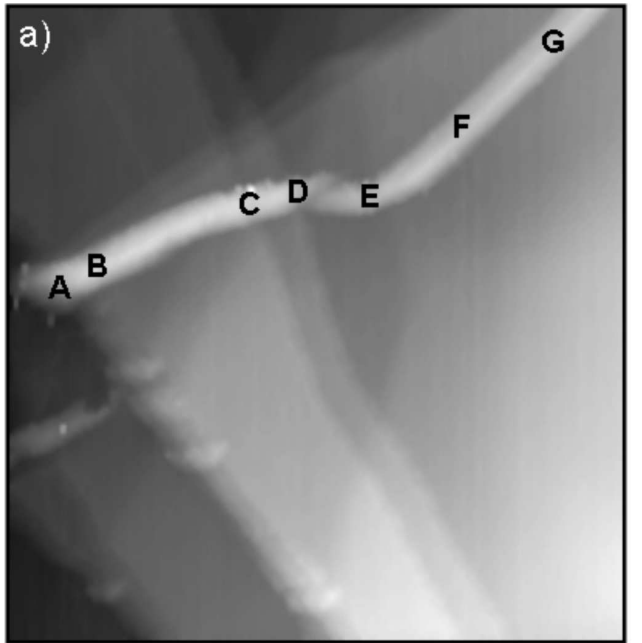

b) current [nA]

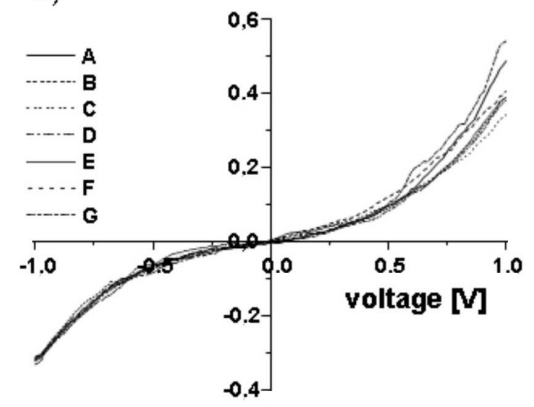

Fig. 2. The image of SWNT $\left(280 \times 280 \mathrm{~nm}^{2}\right)$ along with its highlighted points, for which $I-V$ characteristics were taken.

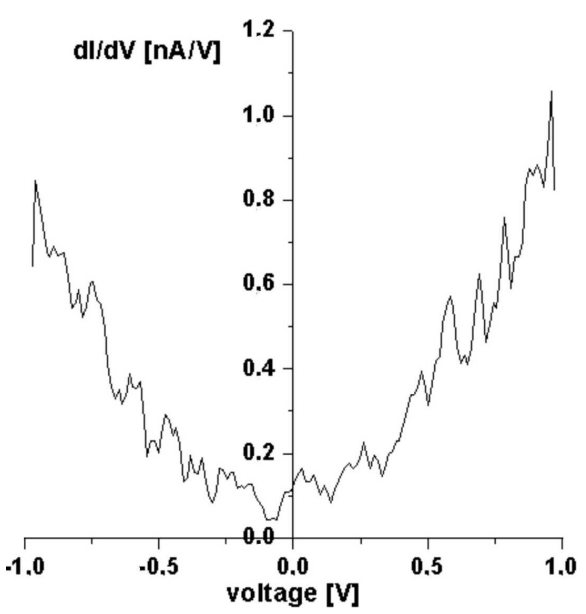

Fig. 3. The dynamic conductance $\mathrm{d} I / \mathrm{d} V=G(V)=\left(1+3 \gamma V^{2}\right)$ of CNT from Fig. 2 .

where $\alpha, \gamma$ - constants. The curves of $I-V$ characteristics are almost identical — insignificant differences occur for the voltage higher than $+0.6 \mathrm{~V}$. The dynamic conductance $\mathrm{d} I / \mathrm{d} V$ taken from the averaged out $I-V$ characteristics is shown in Fig. 3.

The dependence of the second power of voltage $\left(\sim V^{2}\right)$ indicates the parabolic shape of the curve which is tantamount to the fact that the investigated CNT has a metallic character. As shown in Fig. 3, the curve of conductance is altered towards the rising values $G(V)$. The magnitude of this displacement is equal to the $\alpha$-constant. 


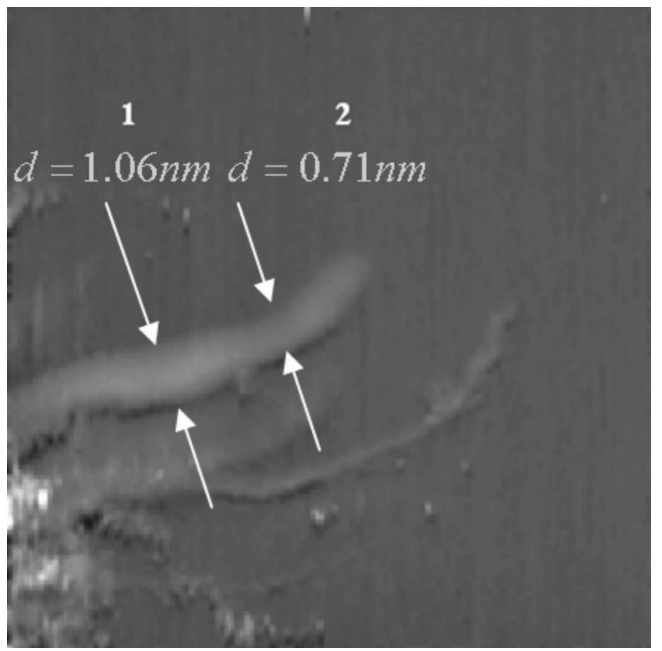

Fig. 4. The STM image of $80 \times 80 \mathrm{~nm}$ area with two cross-sections.

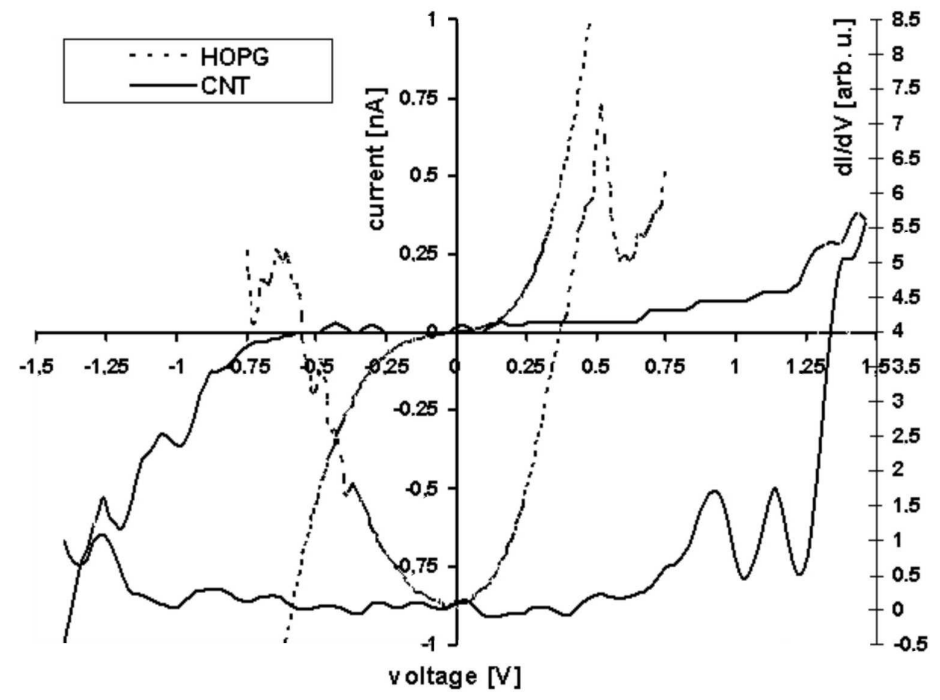

Fig. 5. The $I-V$ characteristics and the dynamic conductivity $\mathrm{d} I / \mathrm{d} V$ in a function of voltage polarization in chosen points of the carbon nanotubes.

Figure 4 shows the topography of SWNT. Its structure is heterogeneous. Its diameter $d$ ranges from $1.06 \mathrm{~nm}(1)$ to $0.71 \mathrm{~nm} \mathrm{(2).} \mathrm{The} \mathrm{objects} \mathrm{located} \mathrm{in} \mathrm{the}$ bottom part of the image are probably the result of additional tunneling of another tip of the same needle (so-called double tip).

Figure 5 shows the comparison of the graphs of $I-V$ and $\mathrm{d} I / \mathrm{d} V$ characteristics of the investigated CNT and $I-V$ and $\mathrm{d} I / \mathrm{d} V$ characteristics of HOPG. The course of the CNT $I-V$ curve reveals its semiconducting character. The en- 
ergy gap estimated on the basis of $I-V$ characteristics amounts to $1.2 \mathrm{eV}$ while differential conductance $\mathrm{d} I / \mathrm{d} V$ oscillates around zero-value within the range of $-0.5 \div 1.0 \mathrm{~V}$. The values of the energy gap for semiconducting CNT may vary depending on object's morphology $(n, m)$.

\subsection{Morphology and diameter measurements of CNTs}

In Fig. 6 another peculiarity in morphology of investigated objects is presented. The arrows show the two CNTs coming out from the inside of other CNTs.

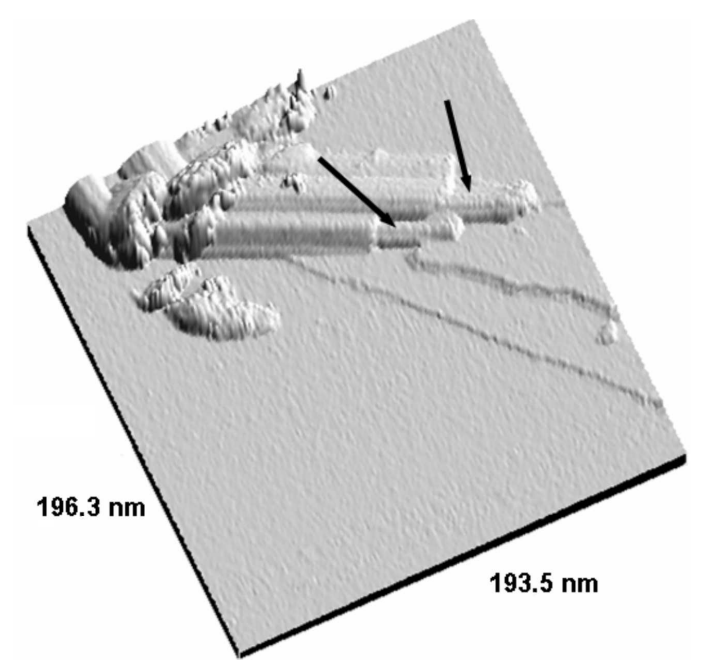

Fig. 6. The image of 3D CNTs coming out from the inside of other CNTs.
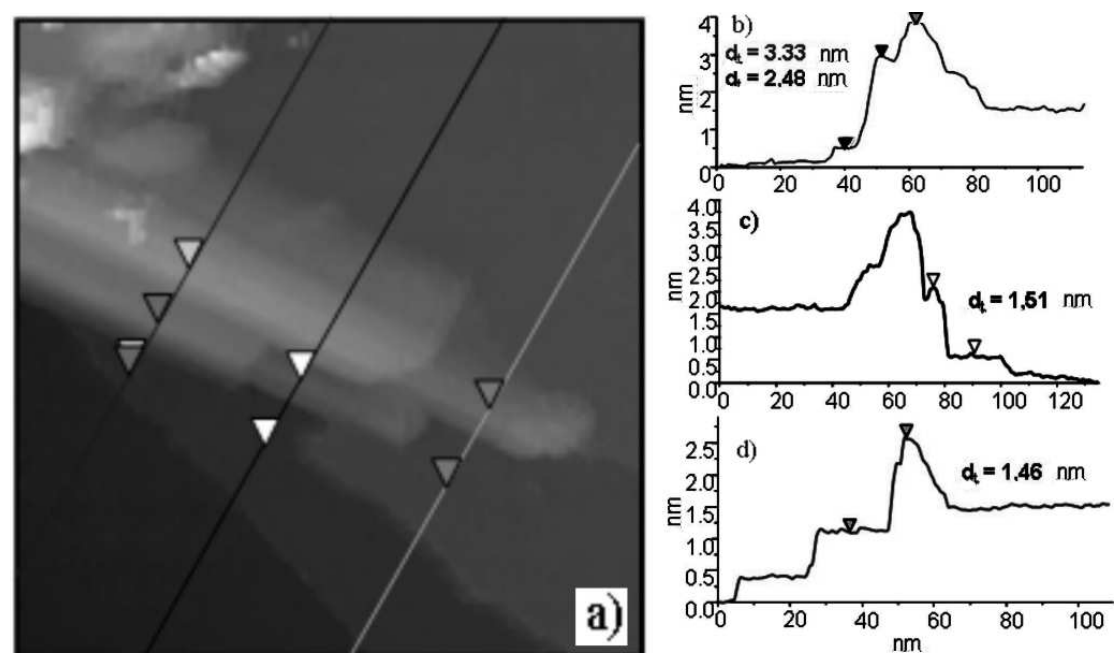

Fig. 7. (a) The image of the CNTs coming out from the inside of other CNTs; (b), (c), (d) other line profiles along with the diameters of the investigated CNTs. 
It is visible in Fig. 7b that the diameters of exterior CNTs differ from one another by about $1 \mathrm{~nm}$. Analyzing the line profiles from Fig. 7c and d it can be noticed that the diameters of the interior CNTs are almost identical. Additionally, knowing that the smallest distances between the MWNTs walls amount to $\approx 0.34 \mathrm{~nm}$ we can assume that between exterior CNT $\left(d_{t}=3.33 \mathrm{~nm}\right)$ and the interior one $\left(d_{t}=1.46 \mathrm{~nm}\right)$ there are another two CNTs. In such case their diameters should amount to $d_{t} \approx 2.14 \mathrm{~nm}$ and $d_{t} \approx 2.82 \mathrm{~nm}$, respectively. However, between CNTs with diameters amounting to $d_{t}=2.48 \mathrm{~nm}$ for exterior diameter and $d_{t}=1.51 \mathrm{~nm}$ for interior one there are no other CNTs.

\subsection{Is it still CNTs or already fullerenes?}

Figure 8 , in which objects with their shape resembling lengthened hemisphere, seems to be interesting.

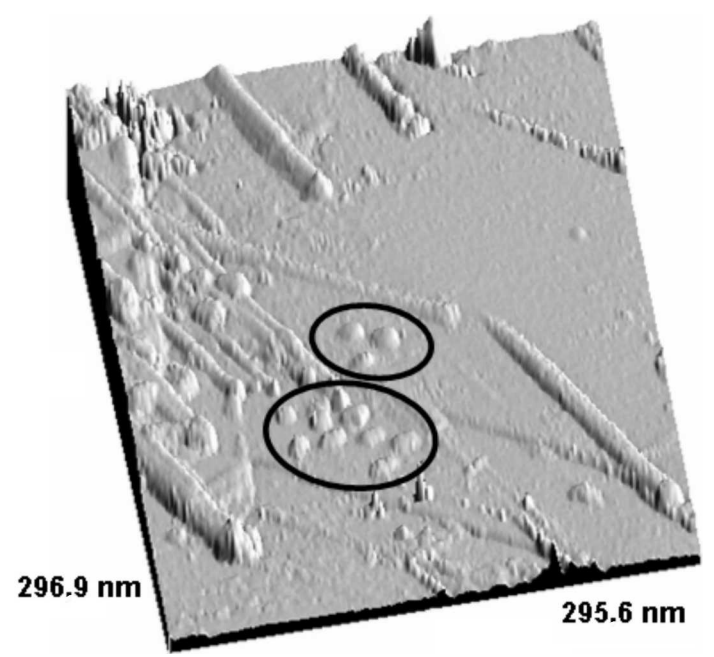

Fig. 8. The 3D image of CNTs. The objects marked with ellipses can be interpreted as short CNTs or fullerenes.

There is a probability that the hemispheres, formed during CNT's synthesis, are fullerenes as the methods of their obtaining are similar to those of CNTs. It is also possible that they are just short CNTs (fullerene C70 is considered to be the shortest SWNT). Figure 9 shows a line profile of the objects from Fig. 8. 

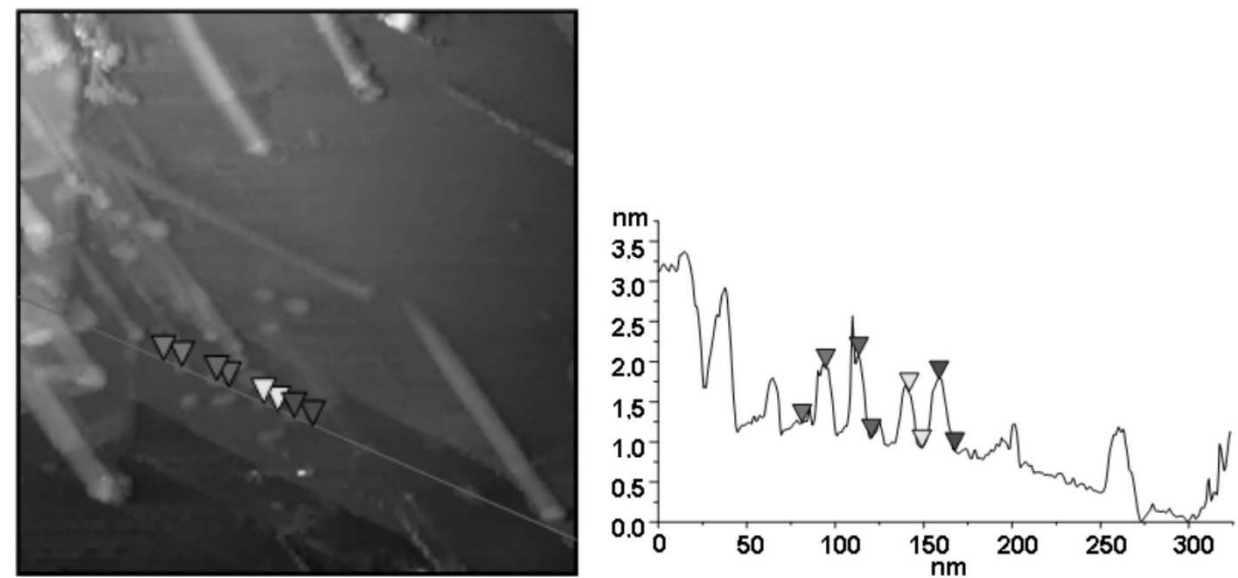

Fig. 9. Image of CNTs $\left(295 \times 295 \mathrm{~nm}^{2}\right)$ along with their line profile. The height of investigated objects marked with triangles amount to approximately: $0.7 \mathrm{~nm}, 1 \mathrm{~nm}$, $0.7 \mathrm{~nm}$, and $0.9 \mathrm{~nm}$, respectively.

\section{Conclusion}

The spectroscopy investigations showed a different conducting character of CNTs, as well as the surface on which they were deposited (HOPG), despite similarities in structure and the character of their bonds. As a result, a considerable influence, which morphology of the investigated materials has on their electric properties, was observed.

Additionally, the conclusions concerning conductivity character and dominance of CNTs of metallic conductivity (at room temperature) are important from the point of view of electronics.

The conclusion presented has crucial implications for CNTs applications. The knowledge of their defects may pose restriction for their applications.

\section{Acknowledgments}

This work was supported by the Poznań University of Technology under PB 62-217/07 BW.

\section{References}

[1] S. Iijima, Nature 354, 56 (1991).

[2] N. Hamada, S. Sawada, A. Oshiyama, Phys. Rev. Lett. 68, 1579 (1992).

[3] J.W. Mintmire, B.I. Dunlap, C.T. White, Phys. Rev. Lett. 68, 631 (1992).

[4] R. Saito, M. Fujita, G. Dresselhaus, M.S Dresselhaus, Appl. Phys. Lett. 60, 2204 (1992).

[5] V. Meunier, M. Buongiorno Nardelli, C. Roland, J. Bernholc, Phys. Rev. B 64, 195419 (2001). 
[6] L.C. Venema, V. Meunier, Ph. Lambin, C. Dekker, Phys. Rev. B 61, 2991 (2000).

[7] J.W.G. Wilder, L.C. Enema, A.G. Rinzler, R.E. Smalley, C. Dekker, Nature 391, 59 (1998).

[8] T.W. Odom, J.-L. Haung, P. Kim, C.M. Lieber, Nature 391, 62 (1998).

[9] Z. Klusek, S. Datta, P. Byszewski, P. Kowalczyk, W. Kozłowski, Surf. Sci. 507510, 577 (2002).

[10] Z. Zhang, C.M. Lieber, Appl. Phys. Lett. 62, 2792 (1993).

[11] C.J. Chen, Introduction to Scanning Tunneling Microscopy, Oxford University Press, Oxford 1993.

[12] R. Wiesendanger, Scanning Probe Microscopy and Spectroscopy Methods and Applications, Cambridge University Press, Cambridge 1994.

[13] D.L. Carroll, P. Redlich, P.M. Ajayan, Phys. Rev. Lett. 78, 2811 (1997).

[14] M. Ishigami, H.J. Choi, S. Aloni, S.G. Louie, M.L. Cohen, A. Zettl, Phys. Rev. Lett. 93, 196803 (2004).

[15] Y.-W. Son, J. Ihm, M.L. Cohen, S.G. Louie, H.J. Choi, Phys. Rev. Lett. 95, 216602 (2005). 